\title{
Variaciones del valor nutritivo del turrón de Jijona en las calidades suprema, extra y popular
}

\author{
Por G. Blázquez Abellán, M. a T. Orzáez Villanueva y A. Díaz Marquina
}

Departamento de Nutrición y Bromatología II: Bromatología. Facultad de Farmacia. Universidad

Complutense de Madrid. Ciudad Universitaria s/n. 28040 Madrid. Teléf. 91/394 18 02. Fax 91/394 1798

\section{RESUMEN}

Variaciones del valor nutritivo del turrón de Jijona en las calidades suprema, extra y popular.

En este trabajo se ha estudiado el valor nutritivo del turrón de Jijona y las variaciones del mismo en las tres calidades tipificadas en la Reglamentación Técnico Sanitaria para Turrones y Mazapanes (Real Decreto 1167/1990). Para ello, se han analizado un total de treinta y tres muestras de turrón de Jijona, divididas de la siguiente forma: veintiuna muestras pertenecen a la calidad Suprema (correspondientes a tres lotes distintos de siete marcas comerciales), nueve a la Extra (tres lotes diferentes de tres marcas comerciales) y tres a la Popular, procedentes de tres lotes de un único fabricante encontrado en el comercio. En todas ellas se ha determinado el contenido de humedad y los macronutrientes: proteína, grasa y carbohidratos. Los resultados y su tratamiento estadístico se muestran en este trabajo.

PALABRAS-CLAVE: Carbohidrato - Grasa - Humedad - Jijona - Proteína - Turrón.

\section{SUMMARY}

Variations on nutritive value of Jijona nougat in suprema, extra and popular qualities.

In this paper, the nutritive value of the three qualities of JijonaNougat has been analysed. These qualities are regulated by the Spanish Regulations for Nougat and Marzipan (Reglamentación Técnico-Sanitaria para Turrones y Mazapanes), (Real Decreto 1167/1990).

Thirty-three samples of Jijona-Nougat have been studied: twentyone samples belonging to "Suprema" quality (corresponding to three different batches of production of seven commercial brands). Nine samples belonging to "Extra" quality (three different batches of production of three commercial brands). Three «Popular» samples, corresponding to three different batches of production from the unique manufacturer found on the market. Moisture and macronutrients: protein, fat and carbohydrates, have been determined in all the samples. The results and their statistical study are shown in this paper.

KEY-WORDS: Carbohydrate - Fat - Jijona - Moisture - Nougat - Protein.

\section{INTRODUCCION}

El turrón de Jijona básicamente es una mezcla de ingredientes naturales de gran calidad: almendra, azúcares, miel y clara de huevo. En su composición principalmente destacan grasas vegetales, glúcidos y proteínas y en menor proporción, si bien bastante importantes, micronutrientes, entre los que destacamos sales minerales y vitaminas, y dentro de éstas las liposolubles $A$ y $E$.

En la actualidad, la fabricación del turrón de Jijona se lleva a cabo principalmente a nivel industrial, debido al gran auge tecnológico alcanzado, lo que permite obtener un producto de extraordinaria calidad, reduciendo al tiempo los costes que supone una elaboración totalmente artesanal, si bien esta última se sigue verificando en reducida escala en fábricas pequeñas de tipo familiar (Pineda, 1992).

Realizada una búsqueda bibliográfica con el fin de encontrar información sobre el turrón de Jijona, nos encontramos con la casi total ausencia de datos sobre este dulce, tan enraizado en nuestras costumbres y fiestas familiares que celebramos en Navidad. Ello fue lo que nos motivó a abrir una línea de investigación sobre este producto, $y$ en trabajos publicados previamente a éste (Blázquez et al., 1995 a), (Blázquez et al., 1995 b), (Blázquez et al., 1996 a), (Blázquez et al., $1996 \mathrm{~b}$ ), hemos realizado un estudio profundo y exhaustivo sobre su origen, composición, elaboración, comercialización y todo lo relativo a la Legislación, incluyendo la Denominación Específica Jijona.

Hemos querido continuar con su investigación, para llegar a obtener un completo conocimiento de este turrón, de excelente valor nutritivo, que en este trabajo ponemos de manifiesto, por lo que el objetivo real del mismo ha sido analizar su composición en macronutrientes y humedad en las cuatro calidades tipificadas en nuestra Legislación sobre Turrones y Mazapanes y que son Suprema, Extra, Estándar y Popular (Real Decreto 1167/1990). 
Nos planteamos, no sólo llegar a obtener un buen conocimiento de este dulce exquisito en distintos tipos de muestras, sino que buscamos aquellas mismas marcas comerciales que los fabricantes ofrecieran en las cuatro calidades mencionadas, con el fin de comparar y poder observar, no solamente la variabilidad de resultados, sino cómo pueden influir los distintos ingredientes básicos utilizados en la elaboración, y comprobar que concordaban con los datos relativos a la Reglamentación ya citada, que los divide en categorías, únicamente atendiendo al contenido de almendra $(64 \%, 50 \%, 44 \%$ y $30 \%$, para las calidades Suprema, Extra, Estándar y Popular respectivamente), componente cuantitativamente prioritario.

\section{PARTE EXPERIMENTAL}

\subsection{Muestras}

En este trabajo se ha realizado un estudio bromatológico en un total de 33 muestras pertenecientes a tres lotes diferentes de siete marcas comerciales, repartidas de la siguiente forma: 21 tabletas corresponden a la calidad Suprema, 9 a la Extra y 3 a la Popular. Asimismo se ha hecho una diferenciación entre las muestras que están acogidas a la Denominación Específica Jijona, y aquellas que no pertenecen a dicha denominación, tal y como se muestra a continuación:

\begin{tabular}{|c|c|c|c|}
\hline 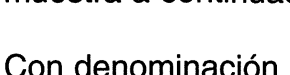 & Suprema & Extra & Popula \\
\hline Específica Jijona & I a V & IV y V & - \\
\hline $\begin{array}{l}\text { Sin denominación } \\
\text { Específica Jijona }\end{array}$ & VI y VII & VI & VII \\
\hline
\end{tabular}

Para poder agrupar e identificar nuestras muestras, atendiendo a las diferentes calidades, marcas y número de lote, se han numerado mediante cuatro dígitos. El primero que aparece es una letra mayúscula (S, E o P), según sea la calidad Suprema, Extra o Popular. El segundo dígito corresponde a un número romano (de I a VII), indicativo de la marca comercial, seguido de un subíndice $(1,2$, ó 3$)$, correspondiente al lote. Por último aparece el número de muestra, que es de 1 a 33. A continuación se especifican las numeraciones para las diferentes calidades:

\begin{tabular}{|c|c|}
\hline Calidades & Muestras \\
\hline Calidad Suprema & S.I $1.1 \mathrm{a} \mathrm{S} . \mathrm{VII}_{3} .21$ \\
\hline Calidad Extra & E.IV $_{1} .22$ a E.VI 3.30 \\
\hline Calidad Popular & $\mathrm{P} . \mathrm{VII}_{1} .31$ a $\mathrm{P} . \mathrm{V} \mathrm{II}_{3.33}$ \\
\hline
\end{tabular}

A la hora de establecer el conjunto muestral se ha tenido una seria dificultad para poder obtener muestras pertenecientes a las cuatro categorías ya comentadas, debido a que los turroneros dedican prácticamente la totalidad de su producción a las calidades Suprema y Extra, y principalmente a la primera; esto fue motivo de la limitación de muestras pertenecientes a la Extra y a la Popular, no encontrando ninguna marca de la Estándar, puesto que no se comercializa; esto ha hecho que no se pudiera obtener una homogeneidad en el número de muestras pertenecientes a estas tres categorías.

Estos turrones se adquirieron en supermercados, hipermercados, almacenes mayoristas y en el caso concreto de los tres lotes correspondientes a la categoría Popular, se obtuvieron en una fábrica de turrón, debido a que es una categoría excepcionalmente comercializada, como se acaba de comentar. El muestreo se ha realizado en la campaña turronera de 1994, entre los meses de octubre a diciembre, que es cuando mayor "stock» de marcas y mayor abundancia de estos productos son ofertados al consumidor.

\subsection{Preparación de las muestras}

El Centro de Investigación y Control de la Calidad, (Ministerio de Sanidad y Consumo, 1985), recomienda para turrones y mazapanes, trocear primero la muestra en pequeños cubos, y pasarlos varias veces por una trituradora eléctrica. Éste sería el procedimiento correcto desde un punto de vista teórico, pero en la práctica se ha podido comprobar que era inadecuado para el turrón de Jijona, por las características típicas de este producto.

Así pues, para obtener una muestra bien homogeneizada se recurrió a rallarla con un cuchillo, a modo de virutas. Una vez así preparada, se guardaba inmediatamente en frascos limpios y secos, de forma que quedasen llenos y se conservaron en un frigorífico. De esta forma se previenen las posibles alteraciones relacionadas tanto con la humedad como con la grasa. Más tarde, ante el problema de espacio, coste y posible rotura de estos frascos en el refrigerador, cambiamos de envase de vidrio a bolsas de plástico, de cierre hermético, de menor precio y mejor manejo. (Ministerio de Sanidad y Consumo, 1985).

\subsection{Metodología analítica y estadística}

Para las distintas determinaciones se ha seguido el método oficial de análisis propuesto en la Legislación para Turrones y Mazapanes.

Para la humedad, en todos los casos se partió entre 5 y 10 gramos de muestra, presecándola en una cápsula de porcelana con arena y etanol, en un baño de agua a una temperatura entre $60-80^{\circ} \mathrm{C}$; más tarde se somete a desecación en estufa convencional a $100{ }^{\circ} \mathrm{C}$ $\pm 2{ }^{\circ} \mathrm{C}$. La determinación de humedad se realizó inmediatamente después de abrir la muestra a fin de evitar posibles modificaciones de este parámetro. (AOAC, 1990).

Referente al contenido en carbohidratos, la mencionada normativa para Turrones y Mazapanes propone la determinación de azúcares reductores, median- 
te el método de Lane-Eynon modificado. En nuestro caso, los azúcares reductores en el turrón de Jijona, no se han podido determinar siguiendo esta metodología, puesto que en repetidos análisis no se obtenían resultados significativos y repetitivos, por consiguiente, se ha recurrido al análisis de los carbohidratos utilizables totales, mediante el método colorimétrico de la antrona de Clegg, puesto que es aplicable a gran cantidad de alimentos y se comprobó que para nuestro producto era el adecuado (Osborne y Voogt, 1986).

El contenido de proteína se determinó tras el previo análisis del Nitrógeno Total determinado por el método Kjeldahl, pesando 1 gramo de muestra con precisión de 0,1 mg.; para el cálculo del porcentaje de proteína bruta se multiplica por un factor de conversión, que para el caso del turrón de Jijona es de 6.25. (Ministerio de Sanidad y Consumo, 1985).

Con relación a la grasa, se pesaron 4 gramos de muestra con aproximación de $1 \mathrm{mg}$., y para facilitar la extracción de la grasa, se llevó a cabo una hidrólisis con $100 \mathrm{ml}$. de ácido clorhídrico $4 \mathrm{~N}$ en caliente, posteriormente se verifica la extracción de la grasa mediante el método de Soxhlet. (AOAC, 1990).

Por último, referente a los tratamientos estadísticos, primero se ha aplicado un análisis de la varianza (Test de Anova), teniendo en cuenta, por un lado, todo el conjunto de nuestros datos y, por otro, por firmas comerciales pertenecientes a las dos categorías mayoritariamente estudiadas: Suprema y Extra; no se ha llevado a cabo ningún tratamiento con la Popular por tener un número de muestras insuficiente y considerarlo no significativo desde un punto de vista estadístico.

Además, se ha aplicado, en concreto, para las marcas comerciales IV, V y VI, que figuran en las dos categorías, primero, un test de Levene para igualdad de varianzas, y a la vista de los resultados obtenidos en éste, dependiendo de que las varianzas hayan sido igual o diferentes, se aplica el test $T$ de Student para varianzas iguales o el test $T$ de Student para varianzas diferentes, respectivamente, para poder comprobar las diferencias o semejanzas que tienen estas marcas en las dos calidades, a las que también se les ha aplicado el test no paramétrico de sumas de rangos (Test de Mann-Withney).

\section{RESULTADOS}

Los resultados analizados en este estudio se han agrupado en un total de 2 tablas y 2 figuras que se detallan a continuación:

La Tabla I refleja el contenido de humedad, así como los valores de proteína, grasa y carbohidratos para la calidad Suprema, Extra y Popular; en ella figuran los datos relativos a los tres lotes que configuran cada marca comercial. Los resultados se expresan en g/100 g de turrón.
La tabla II incluye el tratamiento estadístico aplicado a las marcas comerciales IV, $\mathrm{V}$ y VI pertenecientes a las calidades Suprema y Extra.

En la figura $n . .1$ aparecen los valores medios de las tres calidades analizadas, relativos a los parámetros que han sido objeto de estudio, así como su des-

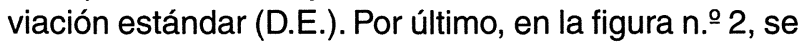
muestran los contenidos medios de humedad y macronutrientes, obtenidos a partir de los tres lotes analizados en cada marca comercial de las tres calidades estudiadas.

Tabla I

Humedad y Macronutrientes: Proteína, Grasa y Carbohidratos Totales en muestras de turrón de Jijona. Calidad Suprema, Extra y Popular. Resultados expresados en $\mathrm{g} / 100 \mathrm{~g}$ de turrón

\begin{tabular}{|c|c|c|c|c|}
\hline \multirow[b]{2}{*}{ Muestras } & \multirow[b]{2}{*}{ Humedad } & \multicolumn{3}{|c|}{ Macronutrientes } \\
\hline & & Proteína & Grasa & Carbohidratos \\
\hline S.I.1. & 3,07 & 15,50 & 38,58 & 40,22 \\
\hline S.I. 2. & 2,71 & 17,06 & 38,30 & 43,67 \\
\hline S.I. 3. & 3,11 & 14,82 & 39,82 & 48,00 \\
\hline S.II. 4. & 3,08 & 15,22 & 37,45 & 43,00 \\
\hline S.II .5 . & 2,73 & 14,83 & 38,50 & 41,35 \\
\hline $\mathrm{S} .11_{3} .6$. & 3,02 & 16,57 & 40,24 & 41,19 \\
\hline S.III 1.7. & 3,04 & 13,34 & 36,64 & 50,84 \\
\hline S.III. 8. & 4,00 & 14,55 & 31,93 & 51,26 \\
\hline S.III .9. & 2,83 & 14,81 & 38,62 & 47,42 \\
\hline S.IV 1.10 & 3,05 & 15,74 & 32,01 & 50,10 \\
\hline $\mathrm{S}^{\mathrm{II}} \mathrm{IV}_{2} 11$ & 3,22 & 16,01 & 34,51 & 46,13 \\
\hline $\mathrm{S}^{\mathrm{IIV}} \mathrm{V}_{3} .12$ & 2,88 & 14,54 & 39,91 & 40,17 \\
\hline$S . V_{1} .13$ & 3,13 & 12,52 & 38,14 & 45,46 \\
\hline S.V $V_{2} .14$ & 3,01 & 15,80 & 37,49 & 44,20 \\
\hline S.V $V_{3} .15$ & 4.31 & 16,27 & 38,96 & 43,31 \\
\hline S.VI .16. & 2,79 & 16.65 & 35,54 & 46,76 \\
\hline S.VI .17 & 2,98 & 16,41 & 38,22 & 40,05 \\
\hline $\mathrm{S} . V I_{3} .18$ & 2,79 & 16,97 & 37,93 & 42,14 \\
\hline S.VII 19. & 3,07 & 15,47 & 35,98 & 46,57 \\
\hline S.VII 20 & 2,94 & 16,21 & 33,67 & 46,78 \\
\hline S.VII .21 & 2,95 & 15,67 & 35,95 & 47,02 \\
\hline E.IV 1.22 & 3,09 & 13,73 & 27,89 & 56,02 \\
\hline E.IV 2.23 & 3,51 & 13,61 & 28,62 & 54,34 \\
\hline$E^{2} I_{3} .24$ & 3,37 & 13,75 & 27,65 & 54,50 \\
\hline E. $V_{1} .25$ & 3,42 & 12,31 & 29,48 & 58,04 \\
\hline E.V ${ }_{2} .26$ & 3,63 & 12,37 & 29,96 & 54,90 \\
\hline E.V. & 2,34 & 13,14 & 31,96 & 49,37 \\
\hline$E . V_{1} .28$ & 3,05 & 13,26 & 30,39 & 62,22 \\
\hline E.VI 2.29 & 2,31 & 12,41 & 30,74 & 64,13 \\
\hline$E . V_{3} .30$ & 3,26 & 12,52 & 27,54 & 59,70 \\
\hline $\mathrm{P} \mathrm{VII} \mathrm{I}_{2} .31$ & 3,48 & 11,79 & 26,83 & 60,35 \\
\hline P.VII 2.32 & 3,35 & 12,17 & 25,99 & 53,11 \\
\hline P.VII 3.33. & 3,37 & 12,02 & 26,04 & 59,85 \\
\hline
\end{tabular}


Tabla II

Valores medios y desviación estándar de humedad y macronutrientes en las marcas IV, V y VI pertenecientes a las calidades Suprema y Extra. Test Estadísticos

\begin{tabular}{|c|c|c|c|c|c|c|}
\hline \multirow[b]{2}{*}{ Parámetros } & \multicolumn{2}{|c|}{ Calidad } & \multicolumn{4}{|c|}{ Test Estadísticos (valores de p) } \\
\hline & $\begin{array}{c}\text { Suprema } \\
(\bar{X} \pm \sigma)\end{array}$ & $\begin{array}{l}\text { Extra } \\
(\bar{X} \pm \sigma)\end{array}$ & $\begin{array}{l}\text { Test de } \\
\text { Levene }\end{array}$ & $\begin{array}{l}\text { Test T de } \\
\text { Student } \\
\text { (Varianzas } \\
\text { iguales) }\end{array}$ & $\begin{array}{c}\text { Test T de } \\
\text { Student } \\
\text { (Varianzas } \\
\text { diferentes) }\end{array}$ & $\begin{array}{c}\text { Test de } \\
\text { Mann- } \\
\text { Withney }\end{array}$ \\
\hline Humedad & $3.1289 \pm 0.47$ & $3.1089 \pm 0.48$ & 0.5942 & 0.9298 & 0.9298 & 0.3309 \\
\hline Proteína & $15.6567 \pm 1.36$ & $13.0111 \pm 0.61$ & 0.2201 & 0.0001 & 0.0002 & 0.0020 \\
\hline Grasa & $36.9678 \pm 2.48$ & $29.3589 \pm 1.54$ & 0.1986 & 0.0000 & 0.0000 & 0.0003 \\
\hline Carbohidratos & $44.2578 \pm 3.26$ & $57.0244 \pm 4.52$ & 0.3400 & 0.0000 & 0.0000 & 0.0005 \\
\hline
\end{tabular}

$\mathrm{p}=$ nivel de probabilidad

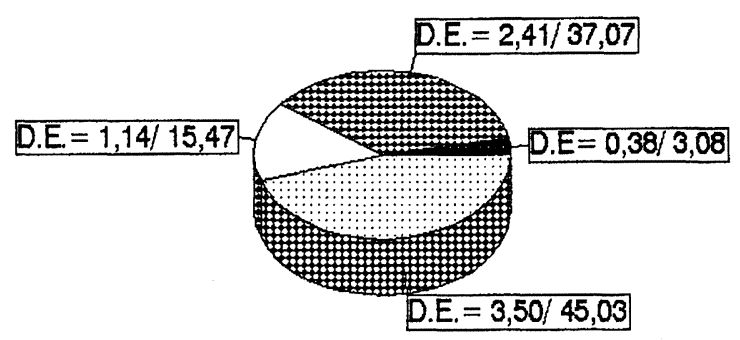

\section{Calidad Suprema}
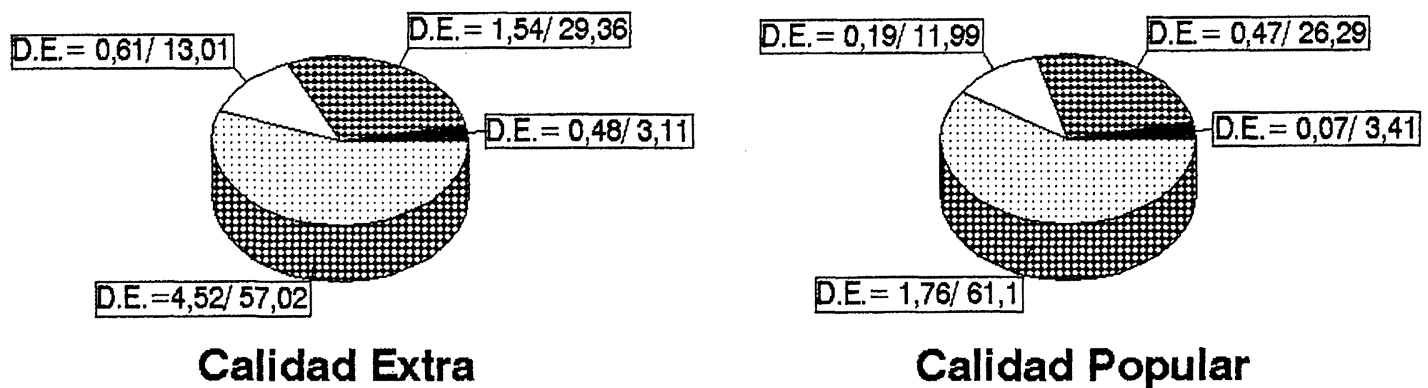

Calidad Popular

W Humedad Grasa $\square$ Proteína $\quad$ Carbohidratos

(D.E. = desviación estándar)

Figura 1

Contenido medio de humedad y macronutrientes en el turrón de Jijona. Resultados expresados en g/100 g de producto. 

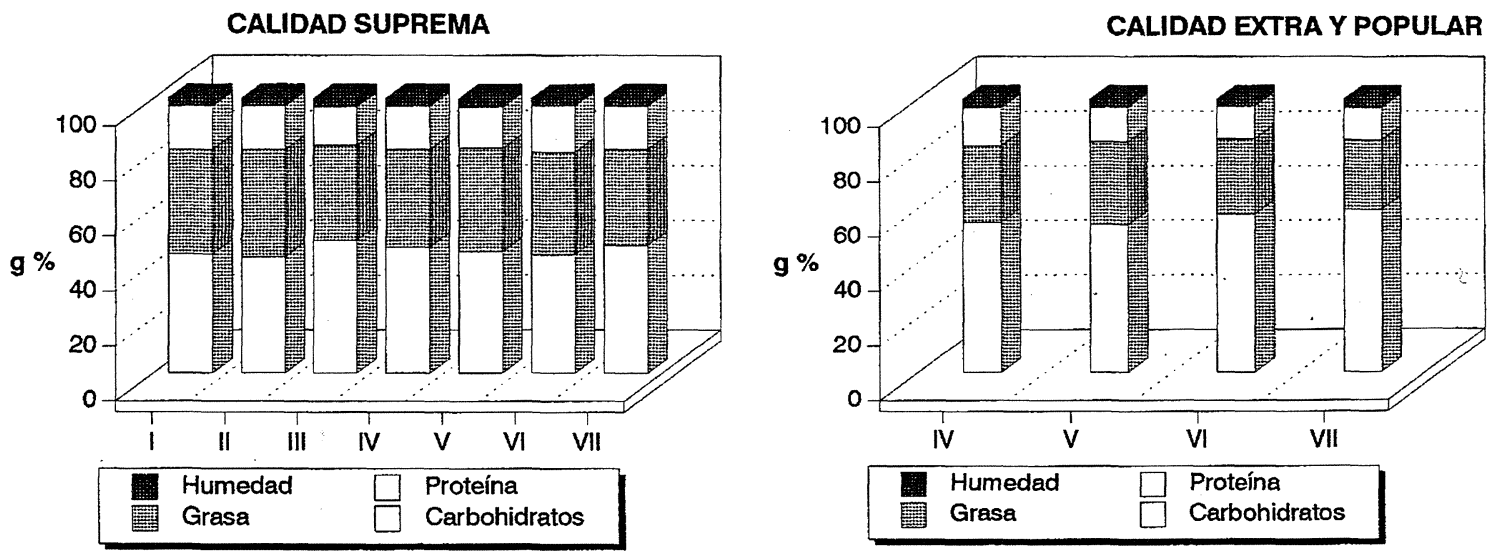

Figura 2

Contenido medio de humedad y macronutrientes en distintas marcas comerciales de turrón de Jijona.

\section{DISCUSIÓN}

La humedad es un parámetro muy interesante a tener en cuenta, ya que puede influir en las características organolépticas de este dulce, relativas a su textura, aspecto, sabor, etc., y en su susceptibilidad a las alteraciones microbianas; por ello, su estudio es importante debido a que interviene en la calidad final del producto y así, en la Reglamentación TécnicoSanitaria para Turrones y Mazapanes, quedan especificados sus contenidos máximos, que difieren según se trate de la calidad Suprema o Extra, y que son de un $4,5 \%$ y $5 \%$ respectivamente para ambas; para la calidad Popular no aparece ninguna especificación relativa a este parámetro.

Los contenidos de humedad aparecen en la tabla I para las tres calidades. Si se tienen en cuenta las 33 muestras de turrón de Jijona analizadas, los contenidos oscilan entre un valor mínimo de $2,31 \%$ para la muestra $E . V_{2} \cdot 29$, y un máximo de $4,31 \%$ para la $\mathrm{S} . \mathrm{V}_{3} .15$; en ningún caso se superan los valores máximos permitidos por la Legislación, anteriormente citados. También existen diferencias entre los tres lotes que configuran una misma marca comercial, así como dentro de cada categoría.

En la figura n. .1 se muestran los contenidos medios de este parámetro en las tres calidades y se observa que éstos son muy similares en las tres categorías, apareciendo la mayor dispersión de resultados en la Extra, igualdad que queda suficientemente avalada por el test de Anova aplicado, con un nivel de significación $\mathrm{p}=0.4459$.

En relación a las marcas que integran cada calidad, a nivel experimental se observa una homogeneidad de resultados, hecho que también se pone de manifiesto mediante el análisis de varianza aplicado, debido a que el nivel de significación es $p=0.4547$ y $p=0.5823$, para las calidades Suprema y Extra respec- tivamente, lo que indica una igualdad, que también queda justificada para las firmas comerciales IV, $\mathrm{V}$ y VI, pertenecientes al mismo fabricante, pero de distinta calidad, Suprema y Extra, tal y como se refleja en la tabla II.

Respecto a los macronutrientes, la grasa es componente mayoritario en el turrón de Jijona, lo que le convierte en un alimento altamente energético. En este caso, la Legislación marca cifras mínimas que son de $34,00 \%$ y $27,00 \%$, para las calidades Suprema y Extra respectivamente, no teniendo en cuenta por completo la Popular.

En la tabla I figuran los contenidos referentes a este macronutriente para las tres calidades. Se observa que los valores, dentro de cada calidąd, no acusan excesivas oscilaciones, si bien existe un descenso en la categoría Extra, que es más acusado en la Popular, en comparación con la Suprema. Esto es lógico, ya que el contenido de almendra difiere en estos tres grupos, tal y como ya se ha comentado (Souci et al., 1994).

Los valores más altos aparecen en la calidad Suprema, y éstos se encuentran entre un $30 \%$ y un $40 \%$, estando casi todos ellos próximos a este último porcentaje, puesto que las marcas que integran este grupo pertenecen a la Denominación Específica del Consejo Regulador Jijona, excepto la VI y la VII, que principalmente utilizan la variedad Marcona y otras muy próximas a ésta en calidad. Además, en esta categoría es donde la Legislación marca mayores contenidos de almendra, un 64\%, a diferencia de la Extra y Popular, en las que establece un $50 \%$ y un $34 \%$ respectivamente, y es este fruto seco el que aporta mayoritariamente este macronutriente. En el caso de la Extra, los contenidos son más similares, y oscilan entre un $27,65 \%$, para la $E . I V_{3} .24$, y un $31,96 \%$, para la $E . V_{3} .27$. Respecto a la categoría Popular, sus valores son bastante homogéneos y próximos a un $26 \%$, aunque se ha de tener en cuenta que únicamente se 
trabaja con tres datos, obtenidos a partir de los triplicados de cada uno de los lotes que integran una única marca comercial.

Lo anteriormente comentado, se puede ver de una forma bastante clara en la figura no 1 , en la que se observa perfectamente el contenido medio de grasa y la desviación standard (D.E) referida a este valor, para las tres categorías. Sometida la totalidad de nuestros datos a un estudio estadístico, se ha comprobado que, con una fiabilidad del $99,99 \%$ se puede decir que existen diferencias entre las tres calidades, hecho que no ocurre cuando se comparan las marcas pertenecientes a cada calidad por separado por los niveles de significación tan elevados que aparecen, $p=0.2443$ y $\mathrm{p}=0.1506$ respectivamente para las calidades Suprema y Extra. Por último, si se comparan las firmas comerciales IV, V y VI, pertenecientes a las categorías Suprema y Extra, al contrario de lo que ocurría con la humedad anteriormente comentada, sí se observa una diferencia total en las mismas en cuanto al contenido en este macronutriente, tanto mediante la aplicación del test $T$ de Student para varianzas iguales, como del test no paramétrico, Mann-Withney.

Los hidratos de carbono son otro de los macronutrientes importantes en el turrón de Jijona, por ser los componentes mayoritarios de su composición centesimal, pero a diferencia de la grasa, éstos son aportados principalmente por la miel y azúcares añadidos en su elaboración, y no por la almendra. También intervienen en dotarle de un alto poder energético, y además en las características organolépticas, sobre todo en lo que respecta al sabor del producto una vez terminado.

El contenido no está tipificado en nuestra Legislación, pero sin embargo, sí existen diferencias importantes dependiendo de la calidad que se trate; básicamente es un parámetro muy dependiente de la formulación, en la que influye, no sólo el contenido de miel, sino el tipo de ésta, y la variabilidad de azúcares que le puedan añadir en su fabricación.

A la vista de los resultados, y en concreto observando la figura $n . \stackrel{0}{1}$, se aprecia un contenido medio creciente desde la calidad Suprema a la Popular; es decir, le ocurre exactamente lo contrario que al otro parámetro ya estudiado, la grasa. Esto es lógico, ya que en la categoría Popular, que es donde aparece en mayor cantidad, también es en la que principalmente utilizan tanto mieles (de uno o varios tipos), como azúcares diversos, y además, disminuyen el contenido de almendra. Esta diferencia queda sobradamente avalada por el análisis de la varianza donde el nivel de significación es $\mathrm{p}=0.0000$.

Los valores pertenecientes a las marcas comerciales que integran la calidad Suprema son heterogéneos, así como los lotes que componen cada una de ellas, con rangos que oscilan entre un porcentaje superior al $40 \%$ e inferior al $52 \%$, diferencia que no se justifica estadísticamente, $(p=0.0715)$. En la categoría Extra, existe una variabilidad en los resultados pertenecien- tes a las tres marcas que constituyen esta categoría $(p<0.05)$ (Tabla I, Figura $n . \circ 2)$.

Referente a la calidad Extra, es importante reseñar que las marcas IV, V y VI, pertenecientes también a la Suprema experimentan un incremento en este macronutriente, aproximadamente de un $17 \%$ al pasar de una a otra categoría, aumento plenamente justificado a nivel estadístico $(p<0.05)$.

Las proteínas, conjuntamente con la grasa y carbohidratos, configuran la práctica totalidad de la composición centesimal del turrón de Jijona, si bien en una menor proporción que los dos anteriores. Es un nutriente valioso, ya que contribuye cualitativa y cuantitativamente en el valor nutritivo de este dulce navideño, y además, presenta la capacidad de poder formar geles, espumas y emulsiones, por lo que es esencial para las propiedades físicas de este alimento.

En la tabla I, se ve claramente cómo en la calidad Suprema se agrupan valores en torno a un $15.5 \%$; la Extra, $12,4 \%$ y la Popular, muy similar a esta cifra, aunque algo inferior, un $12 \%$. Lo que sí se debe decir, es que todas las marcas pertenecientes a la calidad Extra, así como a la Suprema, cumplen la tasa indicada de proteínas, propuesta en nuestra normativa vigente, y que es un mínimo de $12 \%$ y $9,5 \%$ para la calidad Suprema y Extra respectivamente. En esa misma tabla se observa mayor disparidad de resultados entre los tres lotes que conforman una misma casa comercial, perteneciente a la Suprema, mientras que en la calidad Extra, configurada por las marcas IV, $\mathrm{V}$ y VI, aparecen valores bastante más homogéneos entre los tres lotes que integran cada una de ellas, y asimismo ocurre con la Popular.

En la calidad Suprema es en la que aparecen los porcentajes mayores de este nutriente y los contenidos proteicos son similares en las siete marcas analizadas, $(p=0.2185)$, si bien hay que especificar que en la VI y VII no figura tipificada en su etiqueta la Denominación Específica Jijona.

En la categoría Extra los contenidos proteicos han disminuido y en las mismas marcas pertenecientes a ésta y a la Suprema, IV, V y VI, observamos que se producen unos descensos a nivel experimental de $13,27 \%, 18,87 \%$ y $10,56 \%$, al pasar de una a otra respectivamente, hecho plenamente demostrado estadísticamente, ya que los contenidos en proteínas son significativamente diferentes $(p<0.05)$ para las categorías Suprema o Extra. Por otro lado, también se debe comentar que la calidad Popular presenta cifras prácticamente iguales a la Extra, y se produce también una bajada importante, de un $22,49 \%$ al considerar la marca VII, perteneciente también a la Suprema. (figura n.ำ).

Por último, aplicado el test de Anova a nuestros datos, y teniendo en cuenta el conjunto global de nuestras muestras, con un $99.99 \%$ de fiabilidad se puede afirmar que los contenidos medios proteicos en las tres categorías son totalmente diferentes, hecho que se considera totalmente lógico, ya que dependen 
de la cantidad de ingredientes básicos que el fabricante utilice en la formulación del turrón y de la calidad de los mismos.

\section{CONCLUSIONES}

1. ${ }^{\text {a }}$ A la vista de los resultados, se puede afirmar que, en general, las marcas correspondiente a la calidad Suprema, contienen una mayor homogeneidad en todos sus ingredientes básicos respecto a la Extra y Popular.

2. - Referente a los contenidos medios de humedad, éstos son prácticamente iguales para la categoría Suprema y Extra, 3,08\% y 3,10\% respectivamente, y ligeramente superiores en la calidad Popular, con un $3,40 \%$. Dentro de la Suprema, se aprecia una heterogeneidad para este parámetro entre los tres lotes que configuran una misma marca, variabilidad que disminuye al compararlas entre sí.

3. - Respecto al contenido de proteína y grasa, dado que el porcentaje mayoritario procede de la almendra, es su riqueza en este fruto seco lo que justifica que la calidad Suprema presente los índices más elevados de estos macronutrientes.

4. ${ }^{\text {a }}$ En relación a los hidratos de carbono, ocurre exactamente lo contrario al caso anterior, es decir, se encuentra en mayor proporción en la variedad Popular por la diversidad de azúcares que lleva en su composición, así como por la heterogeneidad de las mieles incorporadas en su elaboración y menor proporción de almendra adicionada.

5. - Todas las muestras analizadas cumplen la Reglamentación Técnico-Sanitaria para la elaboración de Turrones y Mazapanes respecto a los contenidos máximos o mínimos de proteína, grasa, y humedad.

6. ${ }^{\text {a }}$ No se han encontrado diferencias significativas en relación a los parámetros analizados, entre las muestras que pertenecen a la Denominación Específica Jijona y aquellas que no están acogidas a ésta.
7. En relación a las marcas $\mathrm{IV}, \mathrm{V}$ y $\mathrm{VI}$, del mismo fabricante, que figuran tanto en la calidad Suprema como en la Extra, se observan diferencias en relación a los macronutrientes: proteínas, grasa y carbohidratos, pero no en cuanto a humedad.

\section{BIBLIOGRAFÍA}

Association of Official Analytical Chemist (1990). - -Official method of analysis»-. 15 $5^{\mathrm{a}}$ Ed. Ed. Association of official analytical Chemist. Arlington.

Blázquez Abellán, G., Orzáez Villanueva, M. a T., Díaz Marquina, A., (1995 a). -«Turrón de Jijona: elaboración artesanal e industrial»-. Alimentación, Equipos y Tecnología. Noviembre. 47-54.

Blázquez Abellán, G., Orzáez Villanueva, M. a T., Díaz Marquina, A., (1995 b). - «Turrón de Jijona: Origen, historia y valor nutritivo"-. Offarm. Diciembre, 50-56.

Blázquez Abellán, G., Díaz Marquina, A., Orzáez Villanueva, M. ${ }^{a}$ T. (1996 a). - «Turrón de Jijona: Comercialización, venta y consumo»-. Alimentaria. Abril. 117-121.

Blázquez Abellán, G., Orzáez Villanueva, M. a T., Díaz Marquina, A., (1996 b). -«Denominación Específica Jijona»-. Alimentaria. Abril. 123-125.

Ministerio de Sanidad y Consumo (1985). - «Análisis de alimentos. Métodos oficiales y recomendados por el Centro de Investigación y Control de la Calidad»-. Ministerio de Sanidad y Consumo. Madrid.

Osborne, D. R. y Voogt, P. (1986). -«Análisis de los alimentos»-. Ed. Acribia, S. A., Barcelona.

Pineda, G. (1992). -«Turrón, tradición y tentación»Financial Food. 76, 11.

Real Decreto 1167/1990, de 21 de septiembre del Ministerio de Relaciones con las Cortes y de la Secretaría del Gobierno, por el que se modifica el Real Decreto 1787/1982, de la Presidencia del Gobierno de 14 de mayo, por el que se aprueba la Reglamentación TécnicaSanitaria para la elaboración y venta de turrones y mazapanes. («B.O.E.» núm. 183, de 2 de agosto de 1982; "B.O.E.» núm. 231 , de 26 de septiembre de 1990).

Rubio, M. J. (1992). - «Sector de turrones, mazapanes y dulces. Año Difícil»-. Aral, 1161: 37-40.

Souci, S. W., Fachmann, W. y Kraut, H. (1994). -«Food Composition and Nutritional Tables»-. Medpharm, Scientific Publishers, Stugttgart.

Recibido: Diciembre 1996 Aceptado: Abril 1997 